\title{
Can echocardiographic assessment of interatrial septum shape and motion improve the accuracy of the BLUE protocol?
}

\author{
ANA OVSENIK ${ }^{1}$, ANDREJ FABJAN ${ }^{2,3}$, MOJCA BERVAR ${ }^{1}$, MATEJ PODBREGAR ${ }^{4,5}$ \\ 1 Department of Cardiology, University Medical Centre, Ljubljana, Slovenia \\ 2 Department of Vascular Neurology and Intensive Care, Neurological Clinic, University Medical Centre, Ljubljana, Slovenia \\ 3 Institute of Physiology, Faculty of Medicine, University of Ljubljana, Slovenia \\ 4 Department of Intensive care, General Hospital Celje, Celje, Slovenia \\ 5 Institute for Pathophysiology, Faculty of Medicine, University of Ljubljana, Slovenia
}

Corresponding author:

Professor Matej Podbregar

Department of Intensive care

General Hospital Celje

Oblakova ulica 5

3000 Celje, Slovenia

phone: +38640215960

e-mail:podbregar.matej@gmail.com

\section{ABSTRACT}

Acute respiratory failure is one of the most challenging critical conditions due to a wide variety of differential diagnosis. Bedside lung ultrasound in emergency (BLUE) protocol allows accurate differentiation between the most common underlying causes of acute respiratory failure in up to $90 \%$ of the cases. The assessment of left atrial pressure affecting left ventricular filling is essential in critically ill patients guiding volume substitution, optimization of left ventricular function and prevention of pulmonary congestion, thus ensuing haemodynamic stability. A simple, non-invasive method of left atrial pressure evaluation is the echocardiographic assessment of interatrial septum shape and motion, which is affected by interatrial pressure gradient. Aiming to improve the accuracy of the BLUE protocol, we propose the simple, non-invasive echocardiographic assessment of interatrial septum shape and motion as an upgrade, providing additional information of the loading of left and right atrium thus distinguishing the most common causes of acute respiratory failure.

Key words: lung ultrasound, BLUE protocol, interatrial septum, echocardiography

\section{INTRODUCTION}

Lung ultrasound (LUS) is a simple, non-invasive, easily repeatable and reproducible bedside diagnostic and monitoring tool, providing real-time dynamic images (1). Compared to conventional thoracic imaging modalities (X ray, CT scan), it is free of radiation hazards and does not require transportation of the unstable patient outside the Intensive Care Unit (ICU) (2). In certain pathologies found in ICU (atelectasis, pneumothorax, pneumonia or acute respiratory distress), LUS has been shown to be more accurate than Chest X-ray, due to the imaging method characteristics itself and suboptimal image quality in supine position of critically ill patients $(3,4)$. Skills needed for the basic LUS assessment can easily be acquired $(5,6)$ and internationally accepted guidelines are now available to assist clinicians with adopting LUS in daily practice (3).

Acute respiratory failure (ARF) is one of the most common and challenging critical conditions presenting to the emergency department or ICU demanding the establishment of the accurate diagnosis in the shortest time possible (7). The variety of differential diagnoses in ARF has led to the development of bedside lung ultrasound in emergency (BLUE) protocol as an extension of the clinical examination, reducing the time needed to diagnosis and directing early therapeutic measures (8). BLUE protocol consists of LUS and venous ultrasound, which enable differentiation between lung oedema, pneumonia, pulmonary embolism, acute exacerbation of asthma/chronic obstructive pulmonary disease and pneumothorax in up to $90 \%$ of the cases (9).

\section{BASICS OF LUS}

Contrary to the traditional belief that ultrasound does not provide the insight into the lung pathology due to high gas content, clinical studies in the past 20 years have proven LUS to be a useful tool in different specialities (10).

Lung ultrasound is typically performed by a convex probe (e.g. abdominal, cardiac, microconvex probe) with scanning of the three standard points on each side, the upper BLUE point, lower BLUE point and postero-lateral alveolar or pleural syndrome (PLAPS) point (11). The easiest way to recognize the correct landmarks for probe positioning is by placing both hands on the patient's chest. The upper hand should lie just beneath the clavicle with finger tips touching the midline and the lower hand below the upper one (Figure 1a). The upper BLUE point is in the middle of the upper hand, while the lower BLUE point is in the middle of the lower palm (Figure 1a). The third point, the PLAPS point, is achieved by the intersection of two lines, a horizontal line running posteriorly from the lower BLUE point and a vertical one presented by the posterior axillary line (Figure 1b). The anterior points of the chest are scanned first, followed by the PLAPS point examination. According to the rules of gravity, air gathers in the upper parts of the lung while fluid accumulates in the basal parts, therefore pleural effusions and lung consolidations are mainly found in PLAPS points (12).

Ultrasound image is formed from the 
echoes generated as ultrasound waves, emitted from the transducer, traverse the tissue. When the ultrasound waves hit the boundary between two tissues with different acoustic properties, a significant proportion of the beam is reflected (13). Due to highly aerated lung parenchyma, the pleural interface reflects nearly all of the transmitted ultrasound, forming a bright echogenic line, with very few waves traversing the interface being absorbed almost immediately (14). Therefore, LUS enables imaging of the lung surface, whereas deeper lung parenchyma is not visualized directly, but through artefacts arising from the lung surface in different pathologies. Nevertheless, LUS enables identification of a variety of pathologies in ARF as most of the cardiorespiratory disorders are superficial and affect the area in close proximity to the pleural line (10).

The interpretation of LUS is based on the recognition of 10 elementary signs, which present non-anatomical images (artefacts) as well as anatomical visualisation of the pleural space and lung parenchyma in the presence of effusion and consolidation (14). With recognition of these basic LUS signs, distinction between normal lung surface (bat sign, A-lines and lung sliding), interstitial syndrome (B-lines), pneumothorax (stratosphere sign and the lung point), pleural effusions (quad and sinusoid sign) and lung consolidations (fractal sign and tissue-like sign) can be made (15). Description of the LUS signs is presented in Table 1, while a detailed explanation with corresponding images can be found in the original article by Lichtenstein et al. (8).

\section{BLUE PROTOCOL}

The principle of the BLUE protocol is division of LUS findings into the following profiles: the A profile associated with anterior lung sliding and A-lines, A' profile with anterior A-lines but without lung-sliding, $B$ profile with anterior lung-sliding and B-lines, B' with anterior B lines without lung sliding, $\mathrm{C}$ profile with anterior lung consolidation and A/B profile as a combination of $\mathrm{A}$ and $\mathrm{B}$ profiles in the lungs (8) (Figure 2).

BLUE protocol starts with determining the pleural line sliding. If the lung sliding is present, we move to the left side of the scheme and anterior LUS points are checked for the A- or B-lines. In case of B-lines, the B profile is found indicating pulmonary oedema, whereas A-lines in the anterior point warrant further differential

Table 1. Description of the basic lung ultrasound signs.

\begin{tabular}{ll}
\hline Lung ultrasound sign & Description \\
\hline bat sign & $\begin{array}{l}\text { hyperechoic pleural line between two adjacent ribs with posterior } \\
\text { acoustic shadowing, where the pleural line represents the belly of the } \\
\text { bat and the ribs its wings }\end{array}$ \\
\hline lung sliding & $\begin{array}{l}\text { the movement of the pleura while breathing, registered on M-mode } \\
\text { as the seashore sign (straight lines presenting the static chest wall } \\
\text { and curved lines presenting the lung surface) }\end{array}$ \\
\hline A-lines & $\begin{array}{l}\text { horizontal hyperechoic artefacts, extending to the bottom of the } \\
\text { screen with a slowly diminishing signal, reflecting the reverberations } \\
\text { of the ultrasound wave between the pleura and the probe }\end{array}$ \\
\hline B- lines & $\begin{array}{l}\text { hyperechoic, well-defined, comet-tail artefacts, arising from the } \\
\text { pleural line, moving along with the lung sliding, extending to the } \\
\text { bottom of the screen and erasing A-lines }\end{array}$ \\
\hline stratosphere sign & $\begin{array}{l}\text { M-mode through the lung surface reveals a stratified pattern due to } \\
\text { absence of lung sliding in case of pneumothorax }\end{array}$ \\
\hline lung sign & $\begin{array}{l}\text { the point where motionless pleura in case of pneumothorax and } \\
\text { normal normal lung surface meet }\end{array}$ \\
\hline quad sign & $\begin{array}{l}\text { quadrangularly shaped anechoic pleural effusion, limited by the } \\
\text { pleural line, lung line and shadows of the ribs }\end{array}$ \\
\hline sinusoid sign & $\begin{array}{l}\text { sinusoid movement of the lung line toward the pleural line on inspi- } \\
\text { ration noted on M-mode }\end{array}$ \\
\hline fractal sign & non-translobar consolidation \\
\hline spleen-like image of the lungs in case of translobar consolidation
\end{tabular}

diagnosis of A profile between pulmonary embolism, pneumonia or acute exacerbation of asthma/COPD. Ultrasound examination of deep leg veins is performed in order to exclude deep vein thrombosis (DVT). When the examination is positive, pulmonary embolism is the most likely diagnosis. When DVT is excluded, PLAPS points are checked for the signs of lung consolidation or effusion. A profile plus positive PLAPS speaks for pneumonia, whereas A profile and negative PLAPS speak for acute exacerbation of asthma/ COPD.

If the lung sliding is absent, we move to the right side of the scheme. In case of B-lines the profile is called B', suggesting pneumonia. The presence of A-lines, however, demands the search of the lung point to establish the final diagnosis of pneumothorax. If the lung point is not found, other diagnostic modalities should be explored. If the $\mathrm{A} / \mathrm{B}$ profile or $\mathrm{C}$ profile is found in the anterior LUS points, no matter the presence or absence of lung sliding, pneumonia is the most likely cause of ARF (8)

\section{ASSESSMENT OF INTERATRIAL SEP- TUM SHAPE AND MOTION}

The assessment of left atrial pressure (LAP) affecting left ventricular filling is essential in critically ill patients guiding volume substitution, optimization of left ventricular function and prevention of pulmonary congestion, thus ensuing haemodynamic stability (16). LAP is most commonly assessed by measurement of pulmonary artery wedge pressure (PAWP), obtained by wedging a pulmonary catheter with an inflated balloon into a small pulmonary arterial branch (17). Due to the invasiveness of the procedure, PAWP measurement has limited utility. Non-invasive estimation of LAP is provided by echocardiography, where E/e' ratio, obtained from mitral inflow Doppler and mitral annulus tissue velocities, correlate well with PAWP $(17,18)$. This correlation is, however, poor in mechanically ventilated and anesthetized patients (19). Furthermore, with limited time available in the emergency setting, a precise echocardiographic examination of diastolic function is inconvenient. LUS in the BLUE protocol allows estimation of extravascular lung water, but is of limited usefulness in prediction of PAWP (14). Therefore, search for new methods of LAP estimation have emerged.

Interatrial septum (IAS) motion recording as a study of hemodynamic relation between atria was first described by Tei in 1979 (20). M-mode of the mid-portion revealed seven characteristic points in IAS motion (20). IAS was found to move 

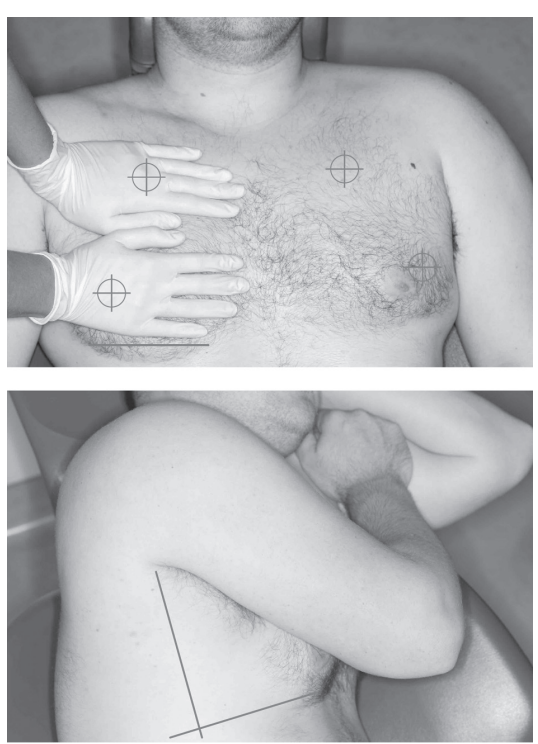

Figure 1. Standard placement of the ultrasound probe in the BLUE protocol. Upper BLUE point and Lower BLUE point (a). PLAPS point (b).

antero-posteriorly during the cardiac cycle, with convex configuration toward the right atrium (RA) in mid- to end-systole and in early-diastole, whereas flat or convex toward the left atrium (LA) in mid- to end-diastole and early systole. (20). With its membranous structure, it is highly dependent on interatrial pressure gradient (IAPG), supported by the findings of limited IAS motion in LA and RA loading situations (20). Mitral stenosis with large leftto-right IAPG was found to be associated with decreased IAS motion throughout the cardiac cycle, with displacement of the IAS towards the RA, while pulmonary hypertension, with increased right-to-left IAPG, was associated with IAS displacement to the left (20). The association between IAS motion and IAPG was further supported by the study in mechanically ventilated patients where IAS was curved towards RA during atrial contraction in end-diastole and in end-systole, but had variable shape in mid-systole depending on the PAWP (21). Mid-systolic reversal, the bowing of the IAS towards LA, was observed during a brief period of passive expiration in patients with a PAWP $\leq 15 \mathrm{mmHg}$, explained by the systemic venous inflow to the RA exceeding the pulmonary venous inflow to the LA. Mid-systolic reversal, observed during passive expiration as well as positive airway pressure inspiration, pointed towards very low PAWP ( $\leq 10 \mathrm{mmHg}$ ). When the PAWP exceeded $15 \mathrm{mmHg}$, IAS remained bowed to the right and midsystolic reversal was absent (21). Similarly, the shape and motion of IAS were found as predictors of change in PAWP in patients undergoing cardiac surgery with marked mid-systolic IAS movement associated with normal- to-low PAWP, mid-systolic reversal associated with normal PAWP and fixed curvature of IAS bowed to the right during the whole cardiac cycle associated with a normal-to-high PAWP (19). Haji et al. found that the fixed curvature of IAS was the best predictor of raised PAWP, when PAWP was $\geq 17 \mathrm{mmHg}$ even in ventilated and anesthetized patients, where Doppler assessment of LAP poorly correlated with PAWP (19). Finally, Masai et al. quantified the IAS motion in AF patients and showed that the amplitude of IAS motion was associated with increased LAP in sinus rhythm and proposed IAS motion as a novel predictor of LAP in AF patients (22).

In the reported studies, transoesophageal echocardiography was used to establish the correlation between shape and motion of IAS and invasive measurement of PAWP. However, in the emergency setting, IAS can be easily viewed by transthoracic echocardiography through any of the available cardiac acoustic windows. Echocardiographic imaging of IAS shape and motion in case of LA and RA loading states are provided in the Illustration 1, Clip 1, Illustration 2 and Clip 2.

The absence of echocardiographic examination in the BLUE protocol was argued by Lichtenstein et al. based on the fact that LUS provides a direct approach to ARF, whereas echocardiography gives indirect arguments (8). Moreover, LUS is claimed to be mastered with far more ease and less time consumption than echocardiography (15). However, the authors suggested that at least a simple cardiac sonography usually follows BLUE protocol in practice (8). The idea of combining LUS with basic echocardiography in diagnosing of acute deterioration of heart failure (ADHF) was already proposed by Russel et al. (23). Previous studies found LUS to be highly sensitive for diagnosing ADHF, but lacking specificity as diffuse B-lines can be seen in many other conditions. By adding echocardiography to LUS, the ADHF was diagnosed with $100 \%$ specificity (23).

Modification of the BLUE protocol has already been proposed by Khosla (24). He suggested diaphragm assessment as an addition to the BLUE protocol arguing that the current protocol does not enable diagnosis of diaphragm paralysis as an underlying pathology in ARF (24). The contra-argument from Lichtenstein et al. was, however, that the protocol should be designed as a simple tool to serve a wide

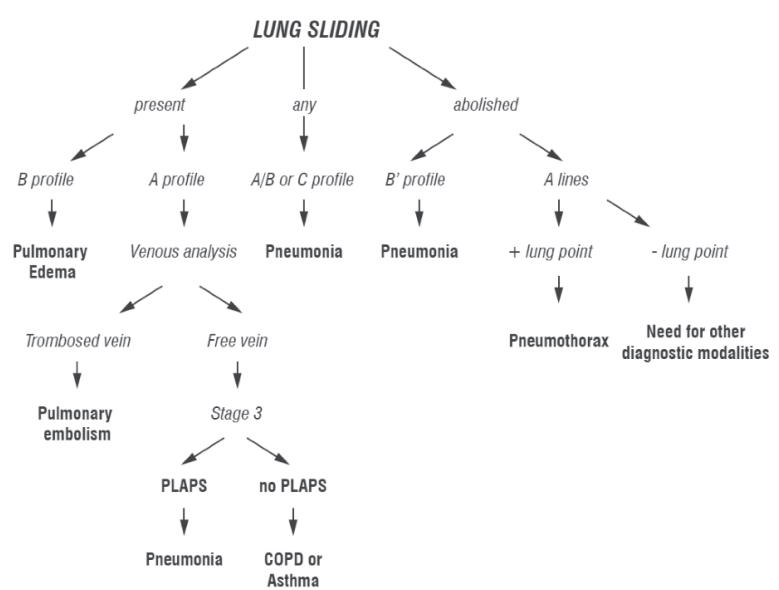

Figure 2. BLUE protocol decision tree (Lichtenstein et al. 2008)

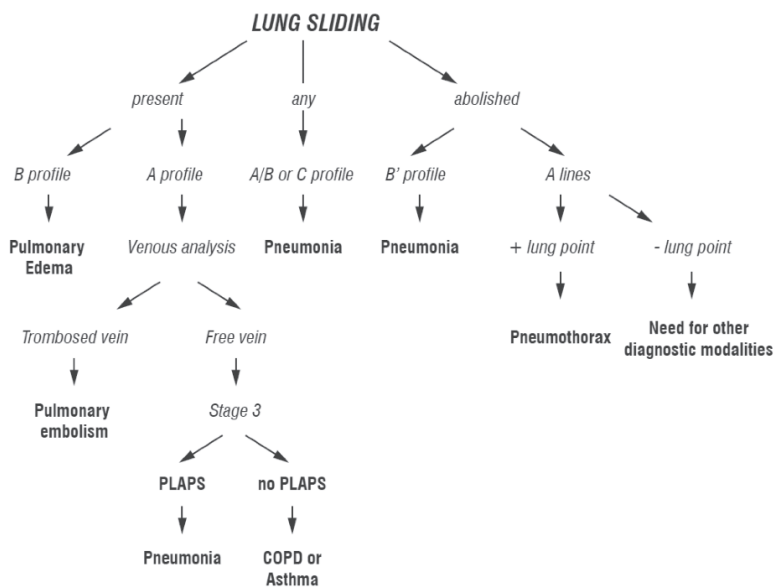

Figure 3. The modified BLUE protocol decision tree. The upgraded protocol allows differentiation between the most common underlying conditions in ARI based on IAS shape and motion. 

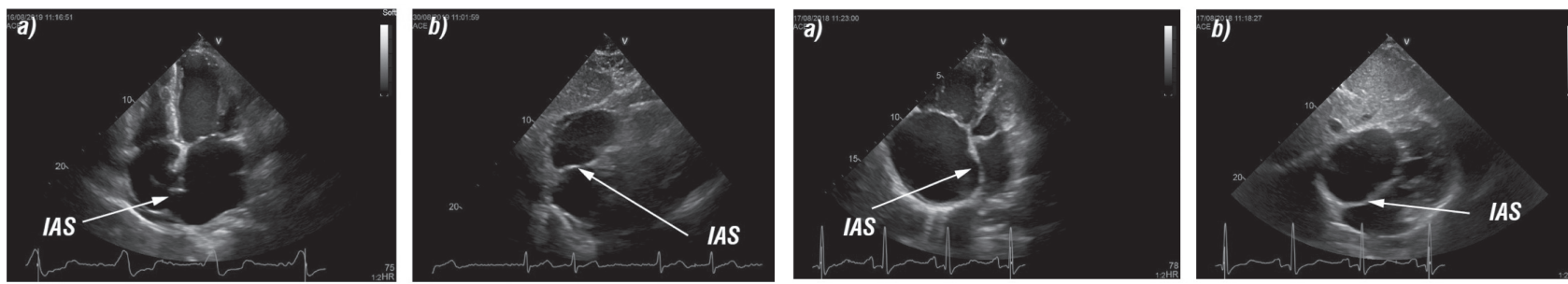

Illustration 1. Four chamber view in a LA loading state (a). Subcostal view in a LA loading state (b).

Illustration 2. Four chamber view in a RA loading state (a). Subcostal view in a RA loading state (b).

a) Available at: https://www.youtube.com/watch?v=wFvtC5Rnjwo

a) Available at: https://www.youtube.com/watch? $v=l J E i w P 6 M 7 y E$

b) Available at: https://www.youtube.com/watch?v=IzWSfTOBt6U

b) Available at: https://www.youtube.com/watch? $v=l D P F k g q f y 2 E$

variety of physicians and address the most common causes of ARF (25).

With that in mind, based on the current knowledge on IAS shape and motion affecting hemodynamic status in critically ill, we propose IAS shape and motion assessment as an addition to the conventional BLUE protocol (Figure 2). The assessment of LAP according to IAS shape and motion is a simple, non-invasive diagnostic method that could more accurately differentiate between the common states predominant- ly loading the LA or the RA with shifting of the IAS to the right in pulmonary oedema and left-sided shifting in pulmonary embolism. The modified version of the BLUE protocol is presented in the following scheme (Figure 3).

\section{CONCLUSION}

No ideal protocol exists just yet that would be as easy as possible to learn, to practice, to interpret, would be performed in just a couple of minutes and would master all the possible pathologies underlying ARF. Aiming to improve the accuracy of the BLUE protocol, we propose the simple, non-invasive echocardiographic assessment of IAS shape and motion as an upgrade, providing additional information of the loading of LA and RA, thus distinguishing the most common causes of ARF.

\section{REFERENCES}

1. Bouhemad B, Brisson H, Le-Guen M, Arbelot C, Lu Q, Rouby JJ. Bedside ultrasound assessment of positive end-expiratory pressureinduced lung recruitment. Am J Respir Crit Care Med 2011;183(3):341-7.

2. Patel CJ, Bhatt HB, Parikh SN, Jhaveri BN, Puranik JH. Bedside Lung Ultrasound in Emergency Protocol as a Diagnostic Tool in Patients of Acute Respiratory Distress Presenting to Emergency Department. J Emerg Trauma Shock 2018;11(2):125-9.

3. Volpicelli G, Elbarbary M, Blaivas M, Lichtenstein DA, Mathis G, Kirkpatrick AW, et al. International evidence-based recommendations for point-of-care lung ultrasound. Intensive Care Med 2012;38(4):577-91.

4. Wooten WM, Shaffer LET, Hamilton LA. Bedside Ultrasound Versus Chest Radiography for Detection of Pulmonary Edema: A Prospective Cohort Study. J Ultrasound Med 2019;38(4):967-73.

5. Strnad M., Zadel S., Klemenc-Ketis Z., Prosen G. Identification of lung sliding: a basic ultrasound technique with a steep learning curve. Signa vitae. 2013;(8)1:31-35.

6. Dexheimer Neto FL, Andrade JM, Raupp AC, Townsend Rda S, Beltrami FG, Brisson H, et al. Diagnostic accuracy of the Bedside Lung Ultrasound in Emergency protocol for the diagnosis of acute respiratory failure in spontaneously breathing patients. J Bras Pneumol 2015;41(1):58-64.

7. Koh Y, Chua MT, Ho WH, Lee C, Chan GWH, Sen Kuan W. Assessment of dyspneic patients in the emergency department using point-of-care lung and cardiac ultrasonography-a prospective observational study. J Thorac Dis 2018;10(11):6221-9.

8. Lichtenstein DA, Meziere GA. Relevance of lung ultrasound in the diagnosis of acute respiratory failure: the BLUE protocol. Chest 2008;134(1):117-25.

9. Lichtenstein D, van Hooland S, Elbers P, Malbrain ML. Ten good reasons to practice ultrasound in critical care. Anaesthesiol Intensive Ther 2014;46(5):323-35.

10. Mojoli F, Bouhemad B, Mongodi S, Lichtenstein D. Lung Ultrasound for Critically Ill Patients. Am J Respir Crit Care Med 2019;199(6):701-14.

11. Lichtenstein D. Novel approaches to ultrasonography of the lung and pleural space: where are we now? Breathe 2017;13(2):100-11.

12. Lichtenstein DA. Lung ultrasound in the critically ill. Ann Intensive Care 2014;4(1):1.

13. Rudas M, Orde S, Nalos M. Bedside lung ultrasound in the care of the critically ill. Crit Care Resusc 2017;19(4):327-36.

14. Goffi A, Kruisselbrink R, Volpicelli G. The sound of air: point-of-care lung ultrasound in perioperative medicine. Can J Anaesth 2018;65(4):399-416.

15. Lichtenstein DA. BLUE-protocol and FALLS-protocol: two applications of lung ultrasound in the critically ill. Chest 2015; 147(6):165970.

16. Volpicelli G, Skurzak S, Boero E, Carpinteri G, Tengattini M, Stefanone V, et al. Lung ultrasound predicts well extravascular lung water but is of limited usefulness in the prediction of wedge pressure. Anesthesiology 2014;121(2):320-7.

17. Peverill RE. "Left ventricular filling pressure(s)" - Ambiguous and misleading terminology, best abandoned. Int J Cardiol 2015;191:1103. 
18. Royse CF, Royse AG, Soeding PF, Blake DW. Shape and movement of the interatrial septum predicts change in pulmonary capillary wedge pressure. Ann Thorac Cardiovasc Surg2001;7(2):79-83.

19. Haji DL, Ali MM, Royse A, Canty DJ, Clarke S, Royse CF. Interatrial septum motion but not Doppler assessment predicts elevated pulmonary capillary wedge pressure in patients undergoing cardiac surgery. Anesthesiology 2014;121(4):719-29.

20. Tei C, Tanaka H, Kashima T, Nakao S, Tahara M, Kanehisa T. Echocardiographic analysis of interatrial septal motion. Am J Cardiol 1979;44(3):472-7.

21. Kusumoto FM, Muhiudeen IA, Kuecherer HF, Cahalan MK, Schiller NB. Response of the interatrial septum to transatrial pressure gradients and its potential for predicting pulmonary capillary wedge pressure: an intraoperative study using transesophageal echocardiography in patients during mechanical ventilation. J Am Coll Cardiol 1993;21(3):721-8.

22. Masai K, Kishima H, Takahashi S, Ashida K, Goda A, Mine T, et al. Interatrial septal motion as a novel index to predict left atrial pressure. Heart Vessels 2018;33(7):762-9.

23. Russell FM, Ehrman RR. A Modified Lung and Cardiac Ultrasound Protocol Saves Time and Rules in the Diagnosis of Acute Heart Failure. J Emerg Med 2017;52(6):839-45.

24. Khosla R. Bedside lung ultrasound in emergency (BLUE) protocol: a suggestion to modify. Chest 2010;137(6):1487; author reply -8. 25. Lichtenstein D., Meziere G. Bedside Lung Ultrasound in Emergency (BLUE) Protocol: Response. Chest 2010;137 (6), 1487-1488. 